\title{
ISOMORPHISMS OF LEXICOGRAPHIC POWERS OF THE REALS
}

\author{
SALMA KUHLMANN
}

(Communicated by Andreas R. Blass)

\begin{abstract}
Given a chain $\Gamma$, we consider the lexicographic order $\mathbb{R}^{\Gamma}$. If $\Delta$ is a chain such that $\mathbb{R}^{\Gamma} \simeq \mathbb{R}^{\Delta}$, we examine the question of whether necessarily $\Gamma \simeq \Delta$. Under an additional hypothesis, we show that $\Gamma$ and $\Delta$ will have the same order types of well ordered subsets. Among other things, this yields an affirmative answer to the above question in the case where $\Gamma$ and $\Delta$ are ordinals.
\end{abstract}

\section{INTRODUCTION}

Let $\Gamma$ be a chain (that is, a totally ordered set), and let $s$ be a mapping from $\Gamma$ into the reals $\mathbb{R}$. Define support $s$ to be the following subset of $\Gamma$ :

$$
\text { support } s=\{\gamma ; s(\gamma) \neq 0\} \text {. }
$$

Consider now the set

$$
\{s ; s: \Gamma \rightarrow \mathbb{R}, \text { support } s \text { is well ordered }\},
$$

and order it lexicographically:

$s_{1}<s_{2}$ if and only if $s_{1}(\gamma)<s_{2}(\gamma)$, for the least $\gamma \in \Gamma$ such that $s_{1}(\gamma) \neq s_{2}(\gamma)$.

This is a well defined total ordering (cf. [FU]), and the totally ordered set thus obtained is called the lexicographic product of the reals with exponent $\Gamma$. We will denote it by $\mathbb{R}^{\Gamma}$. Recall that if $X$ is a well ordered set, the order type of $X$ (in notation o.t. $(X)$ ) is the unique ordinal isomorphic to $X$.

We examine the following problem: if $\Delta$ is any other chain and $\mathbb{R}^{\Gamma} \simeq \mathbb{R}^{\Delta}$ as chains, does it follow that $\Gamma \simeq \Delta$ ? Now given a totally ordered Abelian group

Received by the editors October 4, 1993 and, in revised form, February 7, 1994.

1991 Mathematics Subject Classification. Primary 06A05, 06F20; Secondary 03C60.

Key words and phrases. Lexicograhic products of the reals, order, ordinals, Archimedean complete groups.

This paper represents some results of the author's doctoral thesis.

(C)1995 American Mathematical Society 
$G$, recall that the Archimedean equivalence relation on $G$ is given by

$$
x E y \text { iff } \exists n \in \mathbb{N} \text { s.t. } n|x| \geq|y| \text { and } n|y| \geq|x| .
$$

Recall also that $x$ is said to be infinitely smaller than $y$ (in notation $x \ll y$ ) if and only if

$$
\forall n \in \mathbb{N}: n|x|<|y| \text {. }
$$

Denote the equivalence class of $x \in G$ by $E(x)$. The set $G / E$ of equivalence classes, ordered by

$$
E(y)<E(x) \text { iff } x \ll y,
$$

is a chain with last element $E(0)$; and the order type of the subchain $G / E \backslash$ $\{E(0)\}$ is called the rank of $G$ and denoted $\operatorname{rank}(G)$. It is straightforward to see that the rank is an invariant for isomorphisms of ordered groups. Now if we endow $\mathbb{R}^{\Gamma}$ with pointwise addition, it becomes a totally ordered Abelian group of rank precisely $\Gamma$ (this group is called the Hahn product of the reals with exponent $\Gamma$ ). So the above stated problem is trivial if we assume $\mathbb{R}^{\Gamma} \simeq$ $\mathbb{R}^{\Delta}$ as ordered groups. However, the rank is in general not an invariant for isomorphisms of chains (see the example, due to Charles Holland, at the end of section 2). In section 2, we show that if $\Delta$ is a chain in which $\mathbb{R}$ does not embed, and if $\varphi$ is an embedding of $\mathbb{R}^{\Gamma}$ into $\mathbb{R}^{\Delta}$, then for every ordinal $\alpha$ which is the order type of some well ordered subset of $\Gamma$, there is an element in Image $\varphi$ with support of order type $\alpha$ as well. We deduce from this result that if $\alpha$ and $\beta$ are ordinals such that $\mathbb{R}^{\alpha} \simeq \mathbb{R}^{\beta}$, then $\alpha=\beta$. Other corollaries, shedding light on the problem, are given at the end of section 2 .

In what follows, unless otherwise stated, $\Gamma$ will denote an arbitrary chain. Recall that a subset $I \subset \Gamma$ is an initial segment of $\Gamma$ if and only if, given $\gamma \in \Gamma$ and $i \in I$ such that $\gamma<i$, then $\gamma \in I$. Similarly, a subset $F \subset \Gamma$ is a final segment of $\Gamma$ if and only if, given $\gamma \in \Gamma$ and $f \in F$ such that $\gamma>f$, then $\gamma \in F$. In this article, given $a, b \in\{-\infty, \infty\} \cup \mathbb{R},] a, b[$ will denote the open interval in $\mathbb{R}$ with endpoints $a$ and $b$. The closed interval will be denoted by $[a, b]$, for $a, b \in \mathbb{R}$, and so on for half closed intervals. If $Z$ is a chain and $X, Y$ are subchains of $Z, X \leq Y$ means that for all $x \in X$ and all $y \in Y$ we have $x \leq y$. Similarly for $X<Y$.

At this point, I would like to thank F.-V. Kuhlmann and D. Lascar for many useful discussions.

\section{LEXICOGRAPHIC POWERS WITH ORDINAL EXPONENT}

We need some definitions and lemmas.

Here, $\alpha, \beta, \lambda$ denote ordinal numbers, with $\beta \leq \alpha$. We write $J$ (or $J^{\prime}$ ) to denote a chain in which the chain $\mathbb{R}$ of the reals does NOT embed, and $I$ (or $\left.I^{\prime}\right)$ for an initial segment of $J$.

Let us define

$$
\mathbb{R}_{\beta}^{\alpha}=\left\{s ; s \in \mathbb{R}^{\alpha} \text { and support } s \subseteq \beta\right\} .
$$

If $\beta<\alpha$ and $s \in \mathbb{R}_{\beta}^{\alpha}$ and $a \in \mathbb{R}$, define $\hat{s}^{\wedge} a \in \mathbb{R}_{\beta+1}^{\alpha}$ as follows:

$$
s^{\wedge} a(\lambda)=\left\{\begin{array}{cl}
s(\lambda) & \text { if } \lambda<\beta, \\
a & \text { if } \lambda=\beta, \\
0 & \text { if } \beta+1 \leq \lambda<\alpha .
\end{array}\right.
$$


Suppose now that we have an embedding of chains

$$
\varphi: \mathbb{R}^{\alpha} \longrightarrow \mathbb{R}^{J} \text {. }
$$

We say that $s \in \mathbb{R}^{\alpha}$ is $\beta$-I-determining if for all $s^{\prime} \in \mathbb{R}^{\alpha}$, if $s^{\prime} \uparrow \beta=s \uparrow \beta$, then $\varphi(s) \uparrow I=\varphi\left(s^{\prime}\right) \uparrow I$. Note that:

(1) $\beta=\alpha \Longrightarrow s$ is $\beta$-I-determining, for all $s \in \mathbb{R}^{\alpha}$, and

(2) $\beta<\alpha \Longrightarrow s$ is not $\beta$-J-determining, for all $s \in \mathbb{R}^{\alpha}$.

Lemma 2.1. Suppose that $\beta<\alpha, s \in \mathbb{R}_{\beta}^{\alpha}$ is $\beta$-I-determining and

$$
\text { o.t. (support } \varphi(s) \cap I)=\beta \text {. }
$$

Then there exist $A \subset \mathbb{R}, \operatorname{card} A=2^{\aleph_{0}}$, and $I^{\prime} \supset I$ such that for all $a \in A$, s $^{\wedge} a$ is $(\beta+1)-I^{\prime}$-determining and

$$
\text { o.t. }\left(\operatorname{support} \varphi\left(s^{\wedge} a\right) \cap I^{\prime}\right)=\beta+1 \text {. }
$$

Proof. Let $a \in \mathbb{R}$. Define

$$
j_{a}=\min \left(\left(\operatorname{support} \varphi\left(s^{\hat{\alpha}} a\right)\right) \backslash I\right) .
$$

Note that $j_{a}$ is well defined for all $a \in \mathbb{R}$, except perhaps for only one $b_{0}$ : in fact, suppose $b_{0} \in \mathbb{R}$ is such that support $\varphi\left(s^{\wedge} b_{0}\right) \subset I$; if $a \neq b_{0}$ then necessarily $\varphi\left(s^{\wedge} a\right) \neq \varphi\left(s^{\wedge} b_{0}\right) \quad\left(\right.$ since $\left.s^{\wedge} a \neq s^{\wedge} b_{0}\right)$. But since on the other hand $\varphi\left(s^{\wedge} a\right) \uparrow I=$ $\varphi\left(s^{\wedge} b_{0}\right) \wedge I$, then there exists $j \notin I$ such that $\varphi\left(s^{\wedge} a\right)(j) \neq \varphi\left(s^{\wedge} b_{0}\right)(j)=0$.

Note moreover that since $\varphi$ preserves the order, we have for all $a \in \mathbb{R}$ :

(1) if $\varphi\left(s^{\wedge} a\right)\left(j_{a}\right)>0$, then for all $a^{\prime}>a: j_{a^{\prime}} \leq j_{a}$ and $\varphi\left(s^{\wedge} a^{\prime}\right)\left(j_{a^{\prime}}\right)>0$,

(2) if $\varphi\left(s^{\wedge} a\right)\left(j_{a}\right)<0$, then for all $a^{\prime}<a: j_{a^{\prime}} \leq j_{a}$ and $\varphi\left(s^{\wedge} a^{\prime}\right)\left(j_{a^{\prime}}\right)<0$.

So let $a_{0} \in \mathbb{R}$, and suppose without loss of generality that $\varphi\left(s^{\wedge} a_{0}\right)\left(j a_{0}\right)>0$ (the other case is treated by a symmetrical argument). Hence, for all $a \in\left[a_{0}, \infty[\right.$ we have $\varphi\left(\hat{s}^{\hat{a}} a\right)\left(j_{a}\right)>0$. So consider the decreasing map from $\left[a_{0}, \infty[\right.$ to $J$ which to $a$ associates $j_{a}$. If this map were injective, we would have an embedding of the chain $\mathbb{R}$ in $J$. Hence there exist $\left.a_{1}, a_{2} \in\right] a_{0}, \infty\left[\right.$ such that $a_{1}<a_{2}$ but $j_{a_{1}}=j_{a_{2}}$. Set $j^{\prime}=j_{a_{1}}$ and $I^{\prime}=\left\{j \in J ; j \leq j^{\prime}\right\}$. Let $\left.a^{\prime}, a^{\prime \prime} \in\right] a_{1}, a_{2}[$. Then $j_{a^{\prime}}=j^{\prime}=j_{a^{\prime \prime}}$ (by (1)), moreover for $s^{\prime} \in \mathbb{R}^{\alpha}$ we have:

$$
\left\{\begin{array}{l}
\text { if } s^{\prime} \uparrow \beta+1=s^{\wedge} a^{\prime} \uparrow \beta+1, \\
\text { then } \varphi\left(s^{\prime}\right)(j)=\varphi\left(s^{\prime} a^{\prime}\right)(j)=\varphi\left(s^{\wedge} a^{\prime \prime}\right)(j) \text { for all } j<j^{\prime} .
\end{array}\right.
$$

In fact, if $j \in I$, then it is immediate by hypothesis on $s$, and if $j \notin I$, then by definition of $j^{\prime}, \varphi\left(s^{\wedge} a\right)(j)=0$, for all $a \in\left[a_{1}, a_{2}\right]$. Let us show that then $\varphi\left(s^{\prime}\right)(j)=0$. If not, let $j$ be the least element such that $I<j<j^{\prime}$ and $\varphi\left(s^{\prime}\right)(j) \neq 0$, and suppose first that $\varphi\left(s^{\prime}\right)(j)>0$. But then $s^{\prime} a_{2}>s^{\prime}$, whereas $\varphi\left(s^{\prime} a_{2}\right)<\varphi\left(s^{\prime}\right)$, which is a contradiction. The case with $\varphi\left(s^{\prime}\right)(j)<0$ is treated by a symmetrical argument, by considering $\varphi\left(s^{\wedge} a_{1}\right)$. So assertion (3) holds. Consider now the following subset of $\mathbb{R}$ :

$$
\pi_{a^{\prime}}=\left\{\varphi\left(s^{\prime}\right)\left(j^{\prime}\right) ; s^{\prime} \in \mathbb{R}^{\alpha} \text { and } s^{\prime} \uparrow \beta+1=s^{\wedge} a^{\prime} \uparrow \beta+1\right\} .
$$

Note that $s^{\prime} a^{\prime}$ is $(\beta+1)-\left(I^{\prime} \backslash\left\{j^{\prime}\right\}\right)$-determining (by (3)), and hence $s^{\prime} a^{\prime}$ is $(\beta+1)-I^{\prime}$-determining if and only if $\pi_{a^{\prime}}$ is a singleton. Moreover, $\pi_{a^{\prime}}$ is bounded above in $\mathbb{R}$. To see this, it is enough to observe that if $a^{\prime}<a^{\prime \prime}$ then 
$\pi_{a^{\prime}} \leq \pi_{a^{\prime \prime}}$ : Indeed, assume $s^{\prime \prime} \in \mathbb{R}^{\alpha}$ is such that $s^{\prime \prime} \uparrow \beta+1=s^{\wedge} a^{\prime \prime} \uparrow \beta+1$. But then it is immediate by (3) that

$$
\varphi\left(s^{\prime}\right)(j)=\varphi\left(s^{\prime \prime}\right)(j) \text { for all } j<j^{\prime} .
$$

Since on the other hand $\hat{s}^{\prime} a^{\prime}<\hat{s}^{\prime \prime} a^{\prime \prime}$, we have $s^{\prime}<s^{\prime \prime}$, so $\varphi\left(s^{\prime}\right)<\varphi\left(s^{\prime \prime}\right)$. It follows that $\varphi\left(s^{\prime}\right)\left(j^{\prime}\right) \leq \varphi\left(s^{\prime \prime}\right)\left(j^{\prime}\right)$ as required. Hence let

$$
f:] a_{1}, a_{2}\left[\longrightarrow \mathbb{R}, \quad a \mapsto \sup \pi_{a} .\right.
$$

Then $f$ is increasing. Since $\mathbb{R}$ has the countable chain condition, it follows that $f$ has at most a countable number of discontinuities. Note that $f$ is discontinuous at a point $a$ if and only if $\pi_{a}$ is not a singleton, hence

$$
A=] a_{1}, a_{2}[\backslash\{a ; a \text { is a point of discontinuity of } f\} ;
$$

then $\operatorname{card} A=2^{\aleph_{0}}$, and we have the conclusions of the lemma.

Corollary 2.2. There exists $s^{\alpha} \in \mathbb{R}^{\alpha}$ such that o.t. ( $\left.\operatorname{support} \varphi\left(s^{\alpha}\right)\right)=\alpha$.

Proof. By induction on $\beta \leq \alpha$, we construct $s^{\beta} \in \mathbb{R}_{\beta}^{\alpha}$, and an initial segment $I_{\beta}$ of $J$ such that

(i) $s^{\lambda}=s^{\beta}$ on $\lambda$, and $I_{\lambda} \subset I_{\beta}$ for all $\lambda<\beta \leq \alpha$,

(ii) $s^{\beta}$ is $\beta-I_{\beta}$-determining and o.t. (support $\left.\varphi\left(s^{\beta}\right) \cap I_{\beta}\right)=\beta$.

Set $s^{0}=0$ and $I_{0}=\emptyset$. If $\beta$ is successor, the construction is done using Lemma 2.1. If $\beta$ is limit, set

$$
s^{\beta}=\bigcup_{\lambda<\beta} s^{\lambda} \quad \text { and } \quad I_{\beta}=\bigcup_{\lambda<\beta} I_{\lambda} .
$$

(i) is evident. For (ii), let $s^{\prime} \uparrow \beta=s^{\beta} \uparrow \beta$ and $j \in I_{\beta}$; we show that $\varphi\left(s^{\prime}\right)(j)=$ $\varphi\left(s^{\beta}\right)(j)$. But $j \in I_{\lambda}$ for a $\lambda<\beta$, and $\varphi\left(s^{\beta}\right)(j)=\varphi\left(s^{\lambda}\right)(j)=\varphi\left(s^{\prime}\right)(j)$ (since $s^{\lambda}$ is $\lambda-I_{\lambda}$-determining). For the second assertion of (ii), it is enough to observe that

$$
\text { support } \varphi\left(s^{\beta}\right) \cap I_{\beta}=\bigcup_{\lambda<\beta} \operatorname{support} \varphi\left(s^{\lambda}\right) \cap I_{\lambda} \text {. }
$$

Now given any chain $\Gamma$, we associate to it the following set of ordinals:

$\operatorname{WOT}(\Gamma)=\{\lambda$; there exists a well ordered subset of $\Gamma$ of order type $\lambda\}$.

Note that $\operatorname{WOT}(\Gamma)$ is itself an ordinal, and $\operatorname{WOT}(\lambda)=\lambda+1$. Applying this last corollary, we obtain

Corollary 2.3. Let $\Gamma$ be any chain, and suppose that $\mathbb{R}^{\Gamma}$ embeds in $\mathbb{R}^{J}$. Then $\operatorname{WOT}(\Gamma) \subset \operatorname{WOT}(J)$.

Proof. Let $\lambda \in \mathrm{WOT}(\Gamma)$; then $\mathbb{R}^{\lambda}$ embeds in $\mathbb{R}^{\Gamma}$. By hypothesis it follows that $\mathbb{R}^{\lambda}$ embeds in $\mathbb{R}^{J}$, so by Corollary $2.2, \lambda \in \mathrm{WOT}(J)$, as required.

In particular, this shows that if $J$ is the reverse of an ordinal, then so is $\Gamma$. Another consequence of Corollary 2.2 (applied with $J=\lambda$ ) is

Corollary 2.4. If $\varphi: \mathbb{R}^{\alpha} \longrightarrow \mathbb{R}^{\lambda}$ is an embedding, then there exists an embedding of $\alpha$ in $\lambda$, that is, $\alpha \leq \lambda$. If moreover $\varphi$ is an isomorphism, then $\alpha=\lambda$. 
This last result can be restated as a theorem on ordered Abelian groups in the following way: Let $G_{i}$ be an ordered Abelian group, for $i=1,2$. If $G_{1} \subset G_{2}$, then there is a canonical embedding of $\operatorname{rank}\left(G_{1}\right)$ into $\operatorname{rank}\left(G_{2}\right)$. If this embedding is onto, we say that $G_{1} \subset G_{2}$ is an Archimedean extension. We say that $G_{1}$ is Archimedean-complete if it admits no proper Archimedean extension. Given any chain $\Gamma$, let $\mathbf{H}_{\gamma \in \Gamma} \mathbb{R}$ denote the Hahn product of the reals with exponent $\Gamma$. The following theorem is well known (cf. Fuchs [FU]):

Theorem 2.5. An ordered Abelian group $G$ of rank $\Gamma$ is Archimedean-complete if and only if $G \simeq \boldsymbol{H}_{\gamma \in \Gamma} \mathbb{R}$.

We can now restate Corollary 2.4 as follows:

Theorem 2.6. Suppose that $G_{i}$ is Archimedean-complete and $\operatorname{rank}\left(G_{i}\right)$ is an ordinal, for $i=1,2$. If $G_{1} \simeq G_{2}$ as chains, then $G_{1} \simeq G_{2}$ as ordered groups.

In the next corollaries, which all follow from the proof of Corollary $2.2, \coprod_{J} \mathbb{R}$ denotes the subchain of $\mathbb{R}^{J}$ consisting of those sequences with finite support ( $\coprod_{J} \mathbb{R}$ is called the lexicographic sum).

Corollary 2.7. Let $\lambda$ be an infinite ordinal. Then $\mathbb{R}^{\lambda}$ does not embed in $\amalg_{J} \mathbb{R}$.

Corollary 2.8. Suppose that $\Gamma$ is a chain possessing an infinite well ordered subset. Then $\mathbb{R}^{\Gamma}$ does not embed in $\coprod_{J} \mathbb{R}$.

Corollary 2.9. Suppose that $\Gamma$ is a chain such that $\mathbb{R}^{\Gamma}$ embeds in $\amalg_{J} \mathbb{R}$. Then $\Gamma$ is the reverse of an ordinal.

Corollary 2.10. The chain $\mathbb{R}^{J}$ embeds in $\coprod_{J} \mathbb{R}$ if and only if $\mathbb{R}^{J}=\coprod_{J} \mathbb{R}$, which in turn holds if and only if $J$ is inversely well ordered.

We close this paper by giving the following example, due to Charles Holland, which shows that Theorem 2.6 is not true without the assumption on the ranks.

Example. We claim that $\mathbb{R}^{\mathbb{Z}}$ is isomorphic to $\mathbb{R}^{\mathbb{N}}$. Let $K=\mathbb{R}^{\mathbb{N}}$. We partition $K$ into a sequence $\left\{K_{n}\right\}$ of intervals, each isomorphic to $K$, and indexed by $\mathbb{Z}$ as follows. Let

$$
\begin{aligned}
K_{2 m} & =\left\{\left(m, x_{2}, x_{3}, \ldots\right) \mid x_{i} \in \mathbb{R}\right\}, \\
K_{2 m+1} & =\left\{\left(x_{1}, x_{2}, \ldots\right) \mid x_{i} \in \mathbb{R} \text { and } m<x_{1}<m+1\right\} .
\end{aligned}
$$

Now, we partition $\mathbb{R}^{\mathbb{Z}}$ into a sequence $\left\{J_{n}\right\}$ of intervals indexed by $\mathbb{Z}$ and, again, each isomorphic to $K$. Let

$$
\begin{aligned}
& J_{0}=\left\{\left(\ldots, x_{-1}, x_{0}, x_{1}, \ldots\right) \mid i<0 \Rightarrow x_{i}=0\right\}, \\
& J_{n}=\left\{\left(\ldots, x_{i}, \ldots\right) \mid i<-n \Rightarrow x_{i}=0, \text { and } 0<x_{-n}\right\} \text { for } n>0, \\
& J_{n}=\left\{\left(\ldots, x_{i}, \ldots\right) \mid i<n \Rightarrow x_{i}=0, \text { and } x_{n}<0\right\} \text { for } n<0 .
\end{aligned}
$$

Since $m<n \Rightarrow K_{m}<K_{n}$ and $J_{m}<J_{n}$, and $K_{n} \simeq K \simeq J_{n}$, we obtain $\mathbb{R}^{\mathbb{N}} \simeq \mathbb{R}^{\mathbb{Z}}$ 


\section{REFERENCES}

[FU] L. Fuchs, Partially ordered algebraic systems, Pergamon Press, Oxford, 1963.

Mathematisches Institut der Universität Heidelberg, Im Neuenheimer Feld 288, D69120 HeIDELBERG, Germany

E-mail address: fvk@harmless.mathi.uni-heidelberg.de 